\title{
Improvement over the OLSR Routing Protocol in Mobile Ad Hoc Networks by Eliminating the Unnecessary Loops
}

\author{
Shahram Behzad, Reza Fotohi \\ Department of Computer Engineering, Germi branch, Is lamic Azad University, Germi, Iran \\ E-mail:Sh.Behzad173@Gmail.com:Fotohi.Reza@Gmail.Com \\ Shahram Jamali \\ Computer Engineering Department, University Mohaghegh Ardabili, Ardabil, Iran \\ E-mail:jamali@iust.ac.ir
}

\begin{abstract}
Mobile ad hoc networks are type of wireless networks in which any kind of infrastructure is not used, i.e. there are no infrastructures such as routers or switches or anything else on the network that can be used to support the network structure and the nodes has mobility. The purpose of this paper is to provide a better quality of the package delivery rate and the throughput, that is in need of powerful routing protocol standards, which can guarantee delivering of the packages to destinations, and the throughput on a network. For achieving this purpose, we use OLSR ${ }^{1}$ routing protocol that is a responsive protocol and is currently covered under the IETF ${ }^{2}$ standard (RFC 3626). At this paper, we improved the OLSR routing protocol by eliminating the unnecessary loops, and simulation results demonstrated a significant improvement in the criteria of package delivery rate and throughput.
\end{abstract}

Index Terms - Mobile Ad Hoc Networks, Routing, Routing Protocol, OLSR, Dynamic, Topology

\section{Introduction}

Ad hoc networks are mobile networks that communicate with each other without the need to a central structure [1,2], also, the MANET ${ }^{3}$ networks are specific type of networks without infrastructure. Ad hoc network is a collection of mobile nodes that communicate with each other without any centralized control and infrastructure. If sender and receiver (transceivers) are not in commun ication range with each other, then the packages can be sent to the destination node by using the intermediate nodes. Mobile ad hoc networks can be divided into two categories: structured and unstructured networks. Unstructured network or mobile ad hoc network consists of mobile nodes, and

\footnotetext{
${ }^{1}$ Optimized Link State Routing

${ }^{2}$ Int ernet Engineering T ask Force

${ }^{3}$ Mobile Adhoc Net work
}

exchanges information without using a fixed station. In this type of network, nodes are not only doing administrative duties, but also act as a host. Often, nodes in these networks are moving dynamically. There is no base station or fixed structure in the network configuration. Networks are composed of wireless devices, and besides each other form a network with the ability of self-organization. Since the transmission range of a wire less mediu $m$ is limited, communications in this type of networks depend on the intermediate nodes. Thus, each node in the network also plays the role of a router. In this type of networks, the network topology is constantly changing, because of the mobility of the network nodes. In addition, new nodes may be added to the network at any moment or be removed from the network, or some nodes may turn themselves off. So me of the important characteristics of the ad hoc networks include: open and shared transmission medium, dynamic topology, unlimited battery power, limited processing power, limited transmission range, self-organization, distributed cooperation, and being temporary $[3,4]$. In mobile ad hoc networks, we use the OLSR routing protocol [5] that is known as one of the best active and real-time protocols. In this protocol, all nodes constantly communicate with all destinations by exchanging the protocol messages periodically. Consequently, if there were a necessity in emergencies, they would have precomputed routes and broadcast the topological changes to the other nodes. In this paper, we improved the OLSR routing protocol by eliminating the unnecessary loops, and simulation results show a considerable improvement in the criteria of package de livery rate and throughput. The rest of the paper is organized as follows: section Two describes the related work, section three clarifies the Routing protocols in Mobile Ad Hoc Networks, section four portrays the OLSR routing protocol, section five discusses the proposed method (improving the OLSR protocol), section six draws the simulation environment and talks about the parameters evaluation, section seven illustrates the simulation results and finally, section eight will present the 
conclusion of the present paper. e template, and replace the content with your own material.

\section{Related Works}

OLSR is a routing protocol for mobile ad hoc network that has been presented by the MANET working group in the IETF [6]. This protocol acts responsively. OLSR will harm by high variability that is caused by the topology and nature of the traffic. This suggests that, the service quality characteristics may be affecting the performance of OLSR protocol. In [7], the nodes, based on their existing resources, are divided into three categories of service quality nodes, router nodes and receiver nodes. Nodes declare and report their characteristics to their neighbors by sending "HELLO" massages. Each node can specify the categories of its neighbors by receiving the HELLO message from them. Only the service quality nodes can participate in the service quality routing operation. The OLSR routing method is employed for routing. In [8] this is shown that, the OLSR has capability for the quality of routing. In [8] it is also proven that, the existence of more accurate frequent updating will cause positional information throughout the network. General Electronics Company and colleagues [9], create a model of OLSR protocol based on bandwidth of the status link in terms of service quality criteria. This protocol tries to find routes that have the highest bandwidths in bottlenecks. To provide a better service quality (i.e. to provide the route with optimum bandwidth), it is necessary to broadcast the bandwidth changes to calculate the best route with bandwidth correctly. Collagen and colleagues [9] study the impact of MPR ${ }^{4}$ coverage parameter increasing. In addition, concludes that, when the redundancy of topological data and transmissions increase due to the extensive coverage of MPR, despite having average mobile nodes, too many packages are delivered. The study that was carried out in [9] has been further developed in the research [10]. This research emphasizes studying the effect of MPR coverage parameters and TC redundancy adjustments on the OLSR performance. The above-mentioned research suggests that the delivery level will not be affected by overload that is caused by redundant promotional data. Both references $[9,10]$ emphasize having more detailed information on the network surface and about how it effects on the routing protocol performance. In other words, they study the impact of OLSR protocol parameters adjustment in terms of the network status (i.e. nodes and links) rather than the situational information of nodes and links.

\section{Routing Protocols in Mobile Ad Hoc Networks}

Routing in the suggested algorithm is based on routing established upon demand. Each node has a

\footnotetext{
${ }^{4}$ Multi Point Relays
}

routing table in which the node keeps its own routing information. The routing table contains fields such as the destination node address, the next node address, the sequence number, the distance, the minimu m requested bandwidth; the maximum permitted delay, the stream type and the route validity period. The destination node address field specifies the address of the destination node. The next node address identifies the next node on the route for sending packages to the destination. The sequence number field is used to avoid the routing loops formations and repeated transmissions. As a routing message reaches to a node, if the sequence number of the received message for a specific destination node is greater than the sequence number for that specific node in the routing table, the message will be processed. This simple act will prevent from repeatedly sending of the routing packages and avoid the creation of routing loops in routing packages transmission. The distance field specifies the route length. The minimum requested bandwidth specifies the minimu m a mount of bandwidth required by the stream. This field is required only in cases of service quality streams (flows which require the service quality) and will be processed only when the stream type is of quality service. The maximum permitted delay field determines the maximum tolerable delay for the service quality streams. This field is also used only when service quality streams are being sent. The stream type is determined by the stream type field. This field can have the service quality level or the best effort. This field specifies the type of requested service. The validity period field determines the period in which a route is valid. After passing this period, the route will not be valid no more. If this field receives a package for a destination before the end of validity period, the field will be re-initialized. Routing protocols in mobile ad hoc networks can be divided into two categories of table-based or proactive protocols and need-based protocols, the table-based or proactive protocols are used for periodic updating of the links, the routes information are kept in a table and are used whenever they are needed. However, need-based protocols do not require keeping the routes data, and whenever a route is needed, they start to explore a route based on the source location.

\subsection{Simulation Model}

In this category of protocols, each node keeps one or more tables containing routing information to the other nodes of the network, all nodes update their tables to maintain consistency and to have an up to date view about the network. As the network topology changes, the nodes broadcast updating messages throughout the network. This category of protocols is distinctive by the manner of distribution of information about topology changes throughout the network, and by the number of tables that are required for routing. WRP ${ }^{5}, \mathrm{DSDV}^{6}$,

\footnotetext{
${ }^{5}$ Wireless Routing Protocols

${ }^{6}$ Dynamic Destination-Sequenced Distance-Vector
} 
$\mathrm{FSR}^{7}, \mathrm{HSR}^{8}, \mathrm{GSR}^{9}, \mathrm{ZHLS}^{10}$ and CGSR ${ }^{11}$ are some examples of table based protocols.

\subsection{Need-Based Protocols}

In comparison with table-based routing protocols, in this category of protocols, not all updated routes are stored on each node; instead, the routes will be constructed whenever they are needed. When a source node wants to send one message to a destination, it will request the route discovery mechanisms to find a route to the destination (RREQ). Route remains valid until the destination is available or if is not for the long-term needs. Once a route to the destination is found, the RREP mechanis $m$ sends, in reverse, the route to the source node. CBRP ${ }^{12}, \mathrm{AODV}^{13}, \mathrm{DSR}^{14}$, TORA $^{15}$ ABR ${ }^{16}$ are some examples of need-based protocols.

\section{OLSR Routing Protocol}

OLSR is a routing protocol for mobile ad hoc network that has been presented by the MANET working group in the IETF $[11,13]$. This protocol acts responsively. The network nodes exchange the topology information periodically with each other, thus, the optimal route between any two-network nodes is always present. An optimization that, in comparis on with the other status link protocols, has been done in this protocol is to create the concept of MPR. In this protocol, the network nodes are responsible to select a set of their neighbor nodes as MPR set. This set must be chosen in such a way that covers all nodes, which are distant as two steps from the selected node. This protocol recognizes its neighbors and records their network addresses, measures delays or cost towards its neighbors, and exchanges information by forming a package that represents the whole of the information. It sends these packages to all of the routers and calculates the shortest route to every other router. The OLSR routing protocol has the following features: (1) resends only the MPR control messages, (2) reduces the size of the control messages, (3) reduces the network overload, (4) is one stable protocol, (5) is one proactive protocol, (6) doesn't depend on any central entity, (7) supports the nodes mobility and dynamism, (8) is appropriate for dense networks and (9) OLSR protocol involves several steps: generation of the control packages, sending the packages to other nodes, making the shortest path tree (by using the Dijkstra's algorithm) and generation of the routing table. In OLSR, the MPR (Multipoint

\footnotetext{
${ }^{7}$ Fisheye State Routing

${ }^{8}$ Hierarchical State Routing

${ }^{9}$ Global State Routing

${ }^{10}$ Zone-based Hierarchical Link State

11 Clusterhead Gateway Switch Routing

${ }^{12}$ Cluster based Routing

13 Ad hoc On-demand Distance Vector

14 Dynamic Source Routing Protocol

15 Temporally Ordered Routing Algorithm

${ }^{16}$ Associativity Based Routing
}

Relays) points are firstly identified, these points are the only points in the network, that are allowed to broadcast data packages to reduce the network overload and the amount of control packages transmissions. The first job of OLSR is to identify its neighbors by sending Hello packages to all neighbors around each node, by doing this, each node identifies its surrounding nodes and by using the data that are achieved from each one of nodes, it makes a table that contains relationships of the node with its neighbors [11]. At next step, each of the nodes sends its own data with the sequence number in the form of TC packages to the nearby nodes, but the TC packages transmissions are performed only by the MPR nodes [12]. Hence, all existing nodes in the network have information about existing connections and know how to make contact with each node, and related data are stored in the form of a table for each node. Choosing the best route is carried out through the Dijkstra's algorithm. After this step, each node has a routing table that contains the best routes to reach the nearby nodes. In this condition, the network is becoming stabilized. If the nodes change their locations, the above process will be repeated again and the tables will be updated. Actually, using this mechanis mot only reduces the network control overload, but also results that only a set of links (links between the MPRs and their selectors) is introduced to the network nodes. As a result, because of the control messages that MPRs send through the network, a dynamic topology for routing will be given to the nodes.

\section{Our Proposed Method: Improving the OLSR Protocol is Too Short.}

In our suggested method, when a node sends a package to other nodes within its own radio range, packages will be transmitted by nodes called MPR to the other nodes. Consequently, if the package falls into a loop, then two cases occur; (1) if the package used less number of steps (less than 255 steps) to reach the destination, and the package is IP Header, then we set dynamically its number of steps to zero to give the package the second chance to reach the destination, and (2) if the package used more number of steps (more than 255 steps), and the subjected package isn't IP Header, then we eliminate the package, because, otherwise, many packages will remain in the network and this will cause network traffic, bandwidth occupation, high level of delay in the package delivery, and finally, the reduction of package delivery rate and network payload rate. In our suggested method, by eliminating the unnecessary loops, the package delivery rate (PDR) and the throughput is improved by about 20 percent. In this method, by eliminating the unnecessary loops and setting conditions, we prevented from eliminating these packages and consequently improved the package delivery rate and throughput in the network. 


\section{The Simulation Setup and Evaluation Parameters}

\subsection{The Simulation Model}

Here we emphasize the performance evaluation of OLSR routing protocol by using the NS simulator, that is an object oriented and Discrete Event Driven simulator. The NS ${ }^{17}$ software is generally used for simulation of the local computer networks and wide area networks. We could simulate the OLSR protocol by using the latest version of the NS-allinone-2.35 and patching um-olsr-2.35_v0.8.8 version of the OLSR protocol in NS-allinone-2.35. Although, NS can be implemented on different operating systems, but in this paper, we use the Back Track 5 Linu xoperating system, which is being employed for a number of programming tools with simulation process, to run the simulation of NS-allinone-2.35.For Simulation and its implementation, we should firstly design our network scenario in OTCL language that gives us the output as trace. In addition, we use XGAPH to show the graphs, and finally, we employ the auxiliary (supporting) program of NAM to analyze the performance of nodes and how the packages are being sent and eliminated [13].

\subsection{The Simulation Parameters}

In this simulation, we used a wireless network with 802.11 standards, which is a $1000 * 1000$-simulation environment. According to, we employed 802.11 protocols for the network layer, with node transmission range of $250 \mathrm{~m}$, link bandwidth of $11 \mathrm{Mpbs}$, package size of 512 bytes and simulation time of 200s. We evaluated the OLSR efficiency by storing (keeping) the network pace; stop time and size change (the nu mber of mobile nodes). Table.1 shows a summary of the parameters that have been used in this simulation.

Table 1: Values of parameters for OLSR simulation

\begin{tabular}{|l|l|}
\hline \multicolumn{1}{|c|}{ Simulator } & \multicolumn{1}{c|}{ NS2 2.32 } \\
\hline \multicolumn{1}{|c|}{ Protocol } & \multicolumn{1}{c|}{ OLSR } \\
\hline Simulation duration & $30,40,50,60,70$ \\
\hline Simulation area & $1000 \mathrm{~m}$ X $1000 \mathrm{~m}$ \\
\hline Number of nodes & $20,30,40,50,60$ \\
\hline Transmission range & $250 \mathrm{~m}$ \\
\hline MAC Layer Protocol & IEEE 802.11 \\
\hline Pause time & $200 \mathrm{Sec}$ \\
\hline Maximum speed & $20 \mathrm{~m} / \mathrm{s}$ \\
\hline Traffic type & CBR $($ UDP $)$ \\
\hline Data payload & 512 bytes $/$ packet \\
\hline Packet Size & 256 \\
\hline
\end{tabular}

PDR is the number of packages that are delivered to the destination from the source, divided by the total number of packages in the network. This parameter is also called as "success rate of the protocols":

$$
\text { PDR }=(\text { SendPacketNo / RecievePacketNo }) * 100
$$

Where PDR is the package delivery rate, SendPacketNo is the number of sent packages, and RecievePacketNo denotes the number of received packages.

Throughput: a network can be measured by using the different tools that are available on the different operating systems. This page explains the theory, on which the adjustments of these tools for measurements are based, and the is sues related to these measurements. The reason for measurement of the throughput in networks is that, the people often intend to know about the maximum operational power of data in a connection link or network access as expressed by the unit of bit per second. The measurement of this quantity is commonly carried out by transmitting a large size file from one system to another and calculat ing the required time for complete transmitting or copying of the file. Then, with dividing the file size by that time, the throughput will be achieved in unit of megabit per second, kilobit per second or bit per second. The following formula shows how to calculate the throughput:

$$
\mathrm{X}=\mathrm{C} / \mathrm{T}
$$

Where $\mathrm{X}$ is the throughput, $\mathrm{C}$ is the number of requests that are accomplished by the system, and $\mathrm{T}$ denotes the total time of system observation.

\section{Simulation Results}

In graphs, we presented the results for simulation of performance of the conventional and changed OLSR routing protocol by increasing the number of nodes from 30 to 70 and in times from 20 until 60 in a dynamic topology and with the criteria of package delivery rate and throughput. These graphs show that our suggested method (changed OLSR) demonstrates a better improvement than the conventional OLSR. As it can be seen in the Fig.s 1, 2, 3, 4\& 5, our method, along with increasing the number of nodes, shows a significant improvement in comparison with the conventional OLSR, because it reflects more delivered packages to the destination. In addition, as it can be seen in the Fig.s $6,7,8$, and $9 \& 10$, our method has a higher throughput level than the conventional OLSR, and finally, causes a significant increment in the throughput volume.

\footnotetext{
${ }^{17}$ Network Simulator
} 


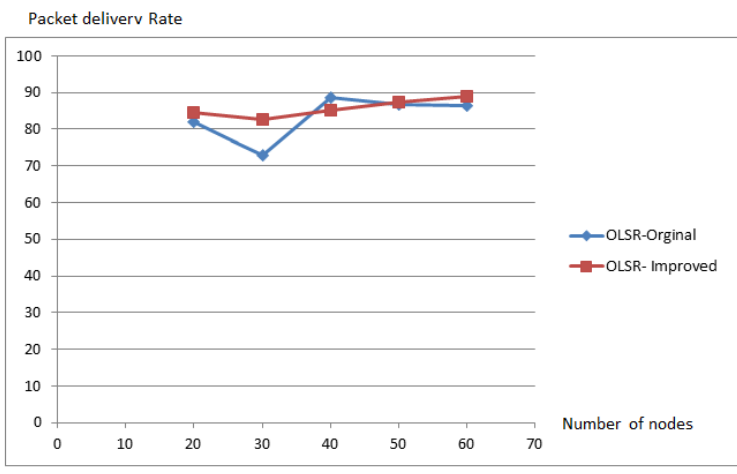

Fig. 1: PDR OLSR Original and OLSR Improved (Duration $=30$ )

Fig. 1 it shows that, the package delivery rate at the time of 30 , when the nu mber of nodes reaches as 40 , the conventional OLSR performs better than our method, but in the other nodes, our imp roved OLSR has the best performance.

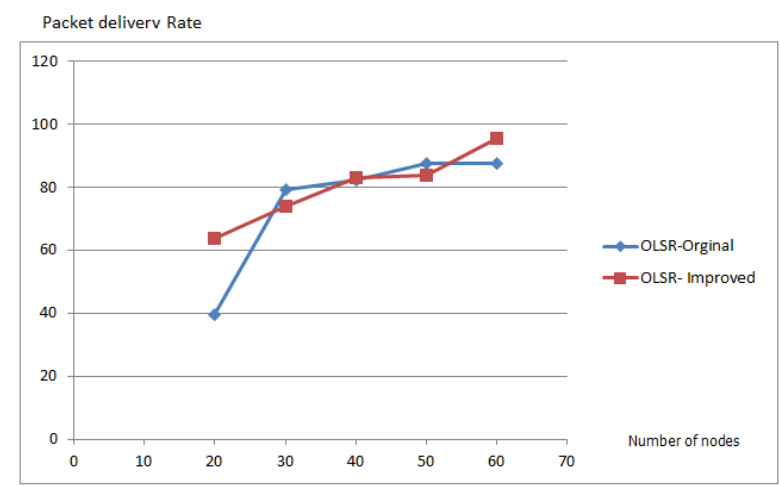

Fig. 2: PDR OLSR Original and OLSR Improved (Duration = 40)

Fig. 2 it shows that, the package delivery rate at the time of 40 , when the number of nodes is 30 to 50 , the conventional OLSR performs better than our method,but in the other nodes,

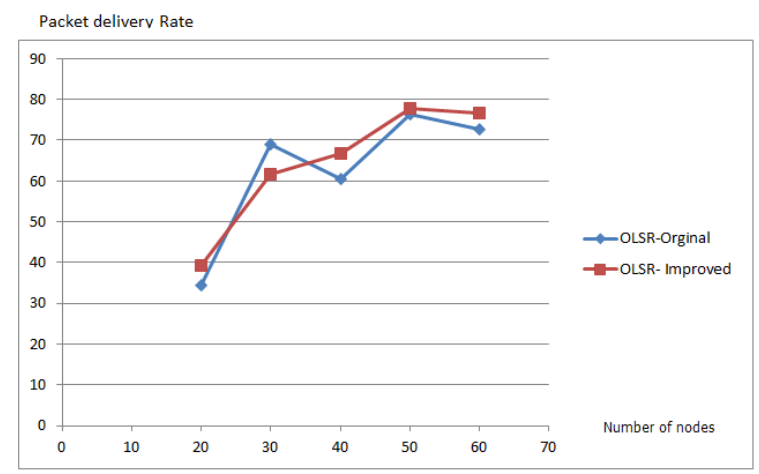

Fig. 3: PDR OLSR Original and OLSR Improved (Duration $=50)$

Fig. 3 it shows that, the package delivery rate at the time of 50, when the number of nodes is 30 , the conventional OLSR performs better than our method, but in the other nodes, our imp roved OLSR has the best performance

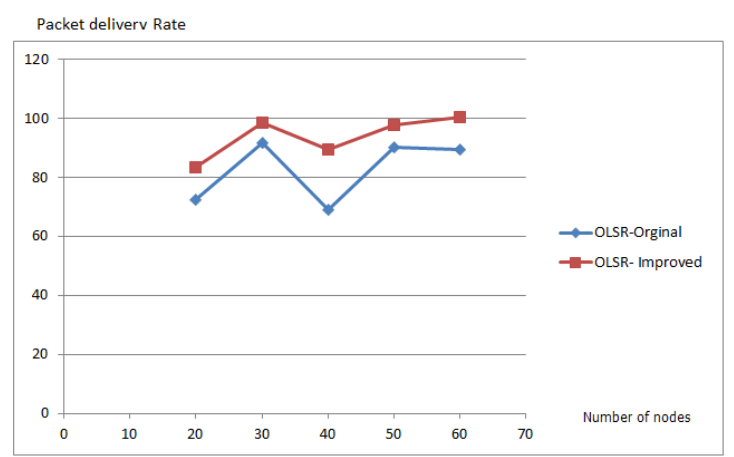

Fig. 4: PDR OLSR Original and OLSR Improved (Duration =60)

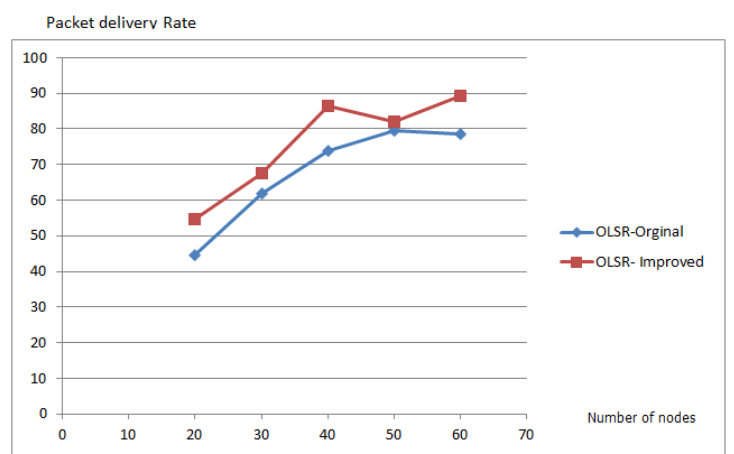

Fig. 5: PDR OLSR Original and OLSR Improved (Duration $=70$ )

Fig. 4 and Fig. 5 they show the package delivery rate at the times of 60 and 70, and for all nodes, our improved OLSR has the best performance towards the conventionalOLSR.

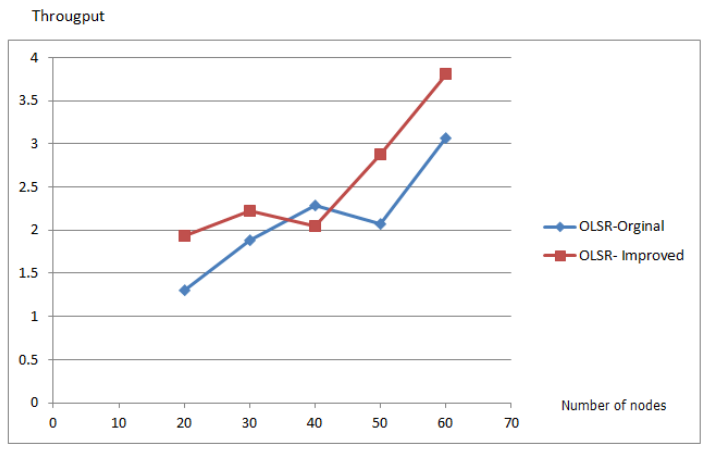

Fig. 6: Throughput OLSR Original and OLSR Improved (Duration = 30)

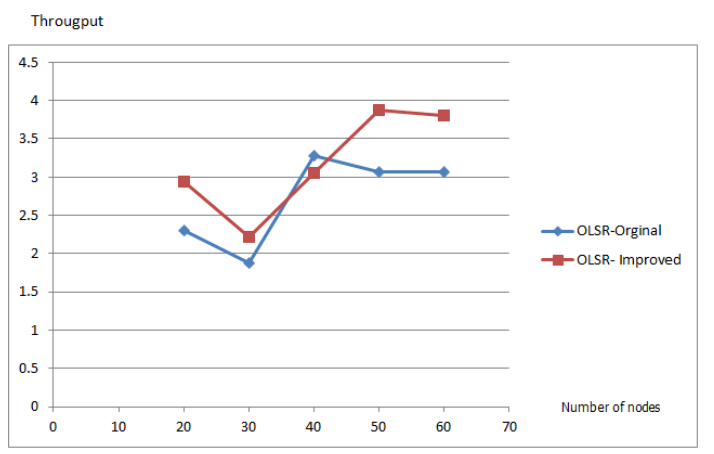

Fig. 7: Throughput OLSR Original and OLSR Improved (Duration $=$ 40) 
Fig. 6 and Fig. 7 they show that, as the nodes number increases, our method has a significant improvement towards the conventional OLSR, because we eliminated the packages, which lasted long in the network and occupied more bandwidth, to free the bandwidth and consequently, the network serviced the other nodes sooner and finally, the total throughput volume increased considerably.

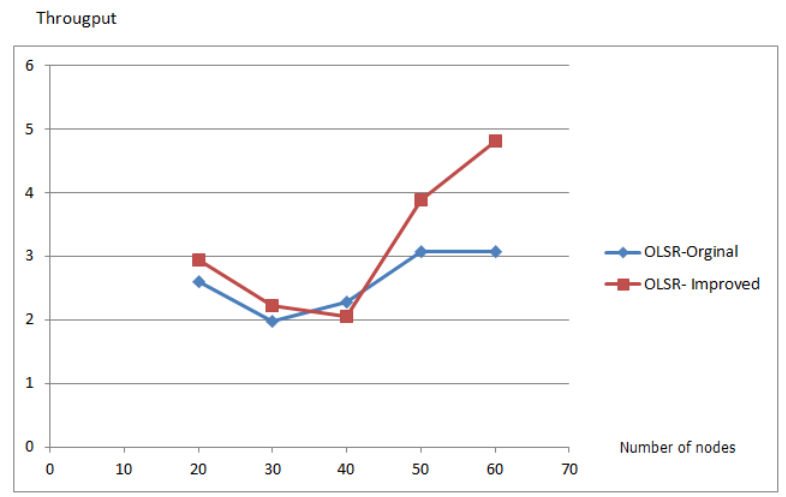

Fig. 8: Throughput OLSR Original and OLSR Improved (Duration = 50)

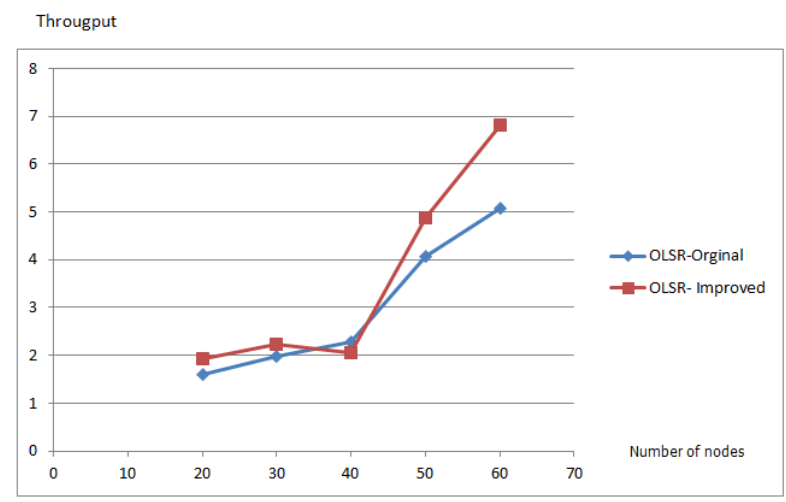

Fig. 9: Throughput OLSR Original and OLSR Improved (Duration = 60)

Fig. 8 and Fig. 9 they show the throughput at the times of 50 and 60 , when the number of nodes reaches as 40 , the conventional OLSR performs better than our method, but in the other nodes, our improved OLSR has the best performance.

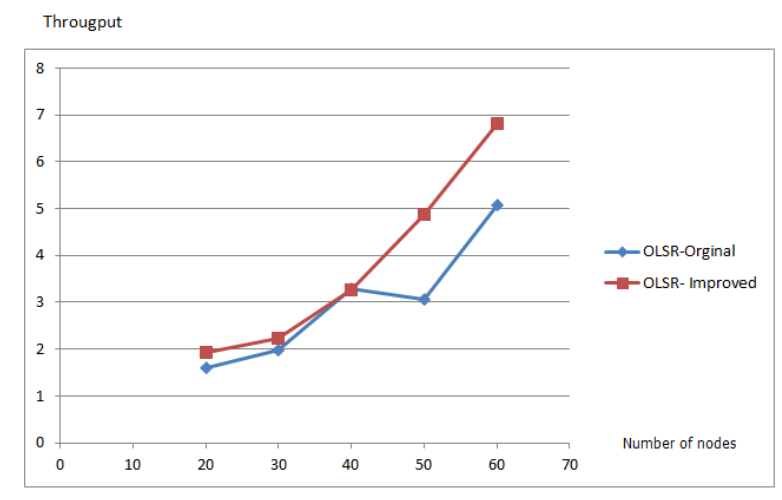

Fig. 10: Throughput OLSR Original and Improved (Duration $=70$ )
Fig. 10 it shows the throughput at the time of 70 , when the number of nodes reaches as 40 , the conventional OLSR has a performance similar to our method, but in the other nodes, our improved OLSR has the best performance

\section{Conclusion}

At this paper, we improved the OLSR routing protocol by eliminating the unnecessary loops. The results of the simulation show that the changed OLSR routing protocol (the suggested method) causes a significant improvement in the criteria of package delivery rate and throughput. The reason for this improvement is that, instead of eliminating all of the packages that fell into the loop, we gave theses package, by setting conditions, the second chance to reach the destination and consequently, in this case, more packages reached to the destination and finally, the package delivery rate and the throughput is improved by about 20 percent in comparison with the conventionalOLSR.

\section{References}

[1] Y. Ge, T. Kunz, L. Lamont, Proactive QoS routing in ad-hoc networks in: Proceedings of the 2nd International Conference on Ad-Hoc Networks and Wireless, Montreal, Canada, October 2003

[2] Ivascu G. L., Pierre S., Qu intero A., "Qos Support based on a Mobile Routing Backbone for Ad Hoc Wireless Networks", IWCMC, Canada, July 2006

[3] T. Clausen, P. Jacquet, Optimized link state routing protocol, IETF RFC3626, 2003.

[4] Li Tingjun, Lin Xueyuan, "GPS/SINS Integrated Navigation System Based On Multi-Scale Preprocessing", Journal of Wuhan University, 2011, Vols 36(1): pp6-9

[5] P.E. Villanueva-Pena, T. Kunz, P. Dhakal, Extending networ knowledge: making OLSR a quality of service conductiveprotocol, in: Proceedings of the International Conference onCommunications and Mobile Computing, Vancouver, Canada, July2006, pp. 103-108

[6] Li Tingjun "Study On Airborne Single passive location Technology" Applied Mechanics and Materials Vols.58 (2011) pp2006-2009

[7] S. Mahfoudh, P. Minet, EOLSR: an energy efficient routing protocol in wire less ad hoc sensor networks, Journal of InterconnectionNetworks 9 (4) (2008) 389-408. 
[8] Li Tingjun "The Phonetic Complex Data Based on FPGA Key Engineering Materials", Vols 475(2011), pp1156-1160.

[9] Hipercom Project, OOLSR Implementation of the OLSR Optimized

[10] T. Clausen, P. Jacquet, L. Viennot, Investigating the impact of partialtopology in proactive MANET routing protocols, in: Proceedings ofthe 5th International Symposium on Wireless Personal MultimediaCommunications, vol. 3, October 2002, pp. 1374-1378.

[11] P.Jacquet, P. Muhlethaler. A. Laouiti, L.Viennot,T. Clauseen, "Optmized Link State Routing Protocol draft-ietf-manet-olsr 05.txt", INTERNETDRAFT,IETF MANET working group.

[12] T. Clausen, P. Jacquet, Optimized link state routing protocol, IETF RFC3626, 2003.

[13] NS -2, The ns Manual (formally known as NS Documentation)

\section{Authors' Profiles}

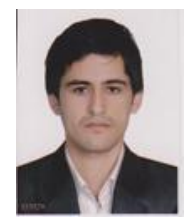

Shahram Behzad: "Msc" Student In Software Engineering Department of Computer Engineering Islamic Azad University Germi Branch,Iran

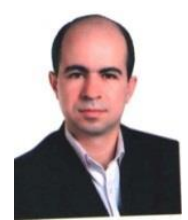

Shahram Jamali: Assistant Proof, Computer Networks and Communication Systems Electrical and Computer Engineering Department University of Mohaghegh Ardabili, Iran

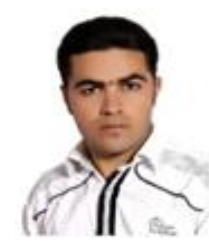

Reza Fotohi: "Msc" Student In Software Engineering Department of Computer Engineering Islamic Azad University Germi Branch,Iran

Shahram Behzad received his B.Sc. in computer engineering from Parsabad University, Parsabad, Iran, in 2008 and his M.Sc. in computer engineering from Azad university of Germi branch, Ardabil, Iran, in 2013.
Shahram Jamali received the B.Sc. degree in electrical and computer engineering from Amirkabir University of Technology, Tehran, Iran, in 1999, the M.Sc. degree in Architecture of Computer Systems from Iran University of Science \& Technology - IUST, Tehran, Iran, in 2001, and the Ph.D. degree in Architecture of Computer Systems from 2008. He is currently associate professor of computer engineering at University of Mohaghegh Ardabili (UMA), His research interests include Congestion control in computer networks, UPFC Controller design, Load balancing in Grid environment.

How to cite this paper: Shahram Behzad, Reza Fotohi, Shahram Jamali,"Improvement over the OLSR Routing Protocol in Mobile Ad Hoc Networks by Eliminating the Unnecessary Loops", International Journal of Information Technology and Computer Science(IJITCS), vol.5, no.6, pp.16-22, 2013. DOI: $10.5815 /$ ijitcs.2013.06.03

Reza Fotohi received his B.Sc. in computer engineering from Shabestar University of Applied Science And Technology, Tabriz, Iran, in 2009 and his M.Sc. in computer engineering from Azad university of Germi branch, Ardabil, Iran, in 2013. His research interests Mobile Ad-Hoc Networks, Performance Evaluation and Optimization algorithms. 